\title{
COMMUNICATIONS
}

\section{Sensitivity Enhancement of a Two-Dimensional Experiment for the Measurement of Heteronuclear Long-Range Coupling Constants, by a New Scheme of Coherence Selection by Gradients}

\author{
Daniel O. Cicero, Gaetano Barbato, and Renzo Bazzo ${ }^{1}$ \\ Istituto di Ricerche di Biologia Molecolare P. Angeletti, Via Pontina km 30.600, 00040 Pomezia, Rome, Italy
}

Received May 18, 2000; revised September 6, 2000

\begin{abstract}
In this work we present a new pulse sequence for the measurement of long-range heteronuclear coupling constants in which the optimization of coherence selection by pulsed field gradients offers a net increase in sensitivity. This type of experiments is extremely valuable for conformational studies of molecules in natural abundance and in this context the use of gradients is essential for an efficient suppression of ${ }^{12} \mathrm{C}$ bound proton signals. A comparative analysis of the different gradient schemes available is presented with a conclusive elucidation of the relative sensitivities. Our gradient scheme could be advantageous as a building block for other related experiments. 02001 Academic Press
\end{abstract}

Key Words: HMBC; long-range heteronuclear couplings; gradient spectroscopy.

Heteronuclear correlations and corresponding coupling constants, especially ${ }^{1} \mathrm{H}-{ }^{13} \mathrm{C}$ coupling constants, are recognized as valuable parameters in assessing the conformation and structure of molecules in solution (1). In the specific case of the conformational analysis of nucleosides in solution, the combined use of ${ }^{1} \mathrm{H}-{ }^{13} \mathrm{C}$ and ${ }^{1} \mathrm{H}-{ }^{1} \mathrm{H}$ couplings greatly improves the level of accuracy that can be obtained for the conformation of the sugar ring, as was already demonstrated in the case of 3'-azido-3'-deoxythymidine (AZT) (2).

A number of different approaches have been used for the measurement of these parameters, including experiments like the HECADE $(3,4)$, HETLOC $(5,6)$, HSQMBC (7), GSQMBC (8-10), HMBC (11-14), and Accordion-optimized experiments (15).

A very convenient way of measuring heteronuclear coupling constants in natural abundance is the use of the two-dimensional heteronucleus-coupled $\omega_{1}$ hetero-half-filtered protonproton correlation (HETLOC) $(5,6)$. A more recent 1D version of this technique was developed in our laboratory (16). These experiments provide coupling constants only for fragments of the type $\mathrm{H}-\mathrm{C}-\mathrm{C}(\mathrm{H})-\mathrm{C}-\mathrm{H}$. On the other hand, experiments

${ }^{1}$ To whom correspondence should be addressed. Fax: 39-06-91093-225. E-mail: bazzo@irbm.it. based on the standard heteronuclear multiple-bond correlation (HMBC) technique (17) are of more general applicability, since they provide values for coupling constants among protons and all classes of carbons, including quaternary carbons.

The first approach to render the original HMBC useful for the measurement of the heteronuclear coupling was described by Titman et al. in 1989 (18). This experiment uses a phasesensitive 2D HMBC pulse sequence (Fig. 1A) and a reference 1D spectrum collected to provide the phase correction necessary for the extraction of the long-range coupling values from the corresponding multiple-bond correlation peaks. Indeed, the internal multiplet structure of such cross peaks is in general rather complex due to a large phase modulation arising from proton shifts and proton-proton coupling evolution during the long fixed delay necessary to build up the multiple-bond correlation. In order to extract the value of such long-range couplings, a fitting procedure has been devized (18), whereby an accurate model is used to represent both the phase modulation and the antiphase structure. In a subsequent paper (19), the authors examined how the accuracy of the coupling constant values measured by this method were affected both by systematic deviations of the data from the theoretical assumptions and by random errors.

The basic assumption of the method described above is the possibility of generating, by calculation from a simple proton spectrum, multiplets with precisely the same phase distortions due to the evolution of proton shifts and couplings as found in the two-dimensional spectrum. More recently, Sheng and van Halbeek have proposed an elegant procedure for obtaining these reference multiplets (12) without the need of any extra data or calculation. They observed that if no suppression of one-bond correlations is performed, one can use the corresponding ${ }^{1} J_{\mathrm{CH}}$ correlation peaks as reference, as the two "satellite" lines have the same phase properties since they are collected under identical experimental conditions.

A second contribution of the authors to this method was the introduction of pulsed field gradients (PFG) to obtain a better suppression of the strong signal arising from protons attached 


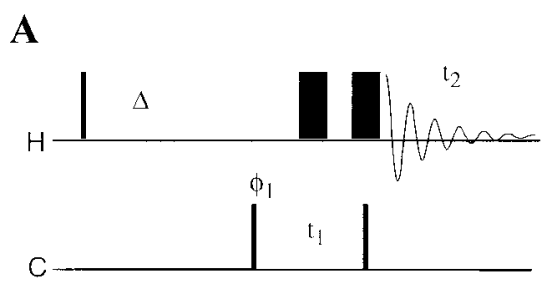

B

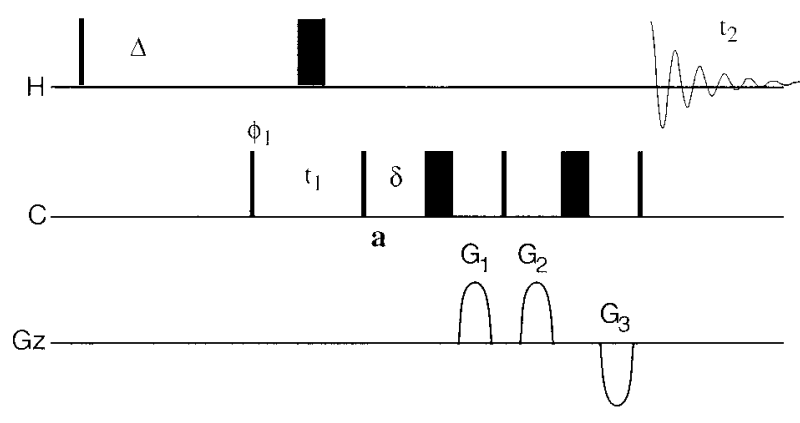

C

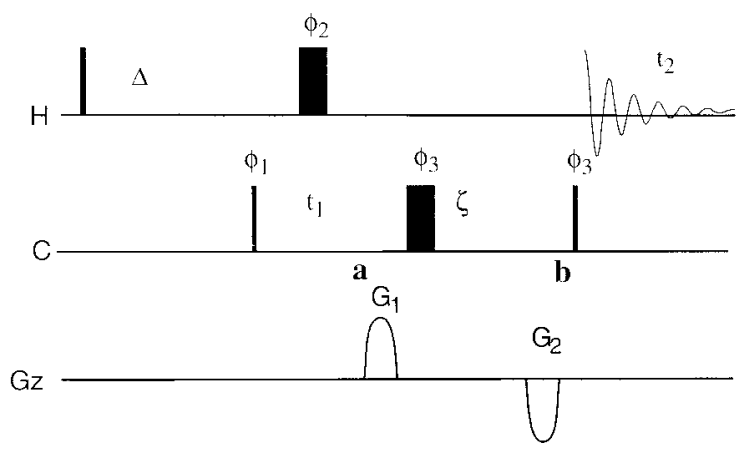

FIG. 1. Pulse sequences of the phase-sensitive HMBC. The delay $\Delta$ is for $\mathrm{H}-\mathrm{C}$ long-range coupling evolution, typically on the order of 40 to $60 \mathrm{~ms}$. (A) Phase-sensitive 2D HMBC, without gradient selection (18). (B) Gradientenhanced 2D HMBC with gradient selection after quadrature detection (12). Quadrature detection is accomplished by incrementing $\phi_{1}$ via the States-TPPI method (29). Delay $\delta$ is calculated to center the subsequent $180^{\circ}$ pulse on $\mathrm{C}$ with respect to the two flanking $90^{\circ}$ pulses. The gradient ratio used is $G_{1}: G_{2}$ : $G_{3}=4: 3:-5$. (C) Phase-sensitive HMBC with echo-antiecho quadrature detection proposed in this work. Even and odd transients are stored in separate memory locations for later recombination to afford fully phase-sensitive data. The following phase cycling is applied: $\phi_{1}=x,-x ; \phi_{2}=x, x,-x,-x$; $\phi_{3}=4 x, 4(-x) ; \phi_{\mathrm{R}}=x,-x, x,-x,-x, x,-x, x$. Gradient ratios: $G_{1}: G_{2}=5:-3$ (odd experiments) and $3:-5$ (even experiments). Delay $\zeta$ is set to guarantee no ${ }^{13} \mathrm{C}$ chemical shift evolution for the first value of $t_{1}$, thereby producing a 0,0 phase correction in $F_{1}$. Its value is calculated as $\zeta=\left(t_{1}\right)_{0}+$ $p_{180 \mathrm{H}}$, where $\left(t_{1}\right)_{0}$ is the initial value of $t_{1}$, and $p_{180 \mathrm{H}}$ is the duration of the proton $180^{\circ}$ pulse.

to ${ }^{12} \mathrm{C}$ nuclei, leading to a significant reduction in $t_{1}$ noise. There are at least three different methods for the use of PFG for the selection of the coherence transfer pathways that involve ${ }^{13} \mathrm{C}$, and produce at the same time pure-absorption lineshapes in the $t_{1}$ dimension. The switched acquisition time (SWAT) method employs gradients during acquisition (20). The second method combines a conventional quadrature detection in $t_{1}$ (for example, using the States-TPPI recipe), with a subsequent gradient selection of only one of the two coherence pathways (21), and it is the one proposed by Sheng and van Halbeek (Fig. 1B). The third method involves the use of gradients also in the quadrature detection, by combining two experiments that select alternatively the $p=1$ or the $p=-1$ coherence pathways. The combination of these two experiments yields a pureabsorption lineshape in $t_{1}(22)$.

Although these two latter gradient selection methods were presented as equivalent (12), the last one proves to be more sensitive. Therefore, on this basis we propose a new pulse sequence (Fig. 1C) that improves by a factor of $\sqrt{2}$ the sensitivity of pulse sequence $1 \mathrm{~B}$.

In the pulse sequence of Fig. 1B, the gradient selection can be considered an addendum to the original phase-sensitive HMBC 1A. It is clear that the intensity of the cosine- or sine-modulated terms of the quadrature in $t_{1}$, generated by changing the phase $\phi$ in the odd or even experiments, respectively, is diminished by a factor of 2 by the gradient selection of only one of the two pathways. This leads to an overall loss in $S / N$ of a factor of 2 when comparing sequences $1 \mathrm{~B}$ and $1 \mathrm{~A}$.

On the other hand, both the cosine- and the sine-modulated terms are detected in the odd or even experiments when pulse sequence $1 \mathrm{C}$ is used (see Appendix), with a reduction by half of their intensity due to the gradient selection. The combination of the two experiments, with a phase shift of $90^{\circ}$ of the even experiments, yields a pure-absorption lineshape in $t_{1}$, where the signal intensity is the same as that in 1A (and thus double with respect to 1B). However, as we obtain this result by summing two experiments, the noise is increased by a factor of $\sqrt{2}$, thereby leading to a reduction of $\sqrt{2}$ in $S / N$ when compared with sequence $1 \mathrm{~A}$, but with an identical gain with respect to sequence 1B. Similar arguments have earlier been proposed for the application of gradients to the three-dimensional HNCO experiment (23).

Two experiments, using sequences $1 \mathrm{~B}$ and $1 \mathrm{C}$, were recorded using identical conditions on a solution of AZT in DMSO as test sample. Figure 2 shows the 2D spectrum obtained using sequence $1 \mathrm{C}$, and the arrow indicates the trace extracted to compare the relative sensitivity of the two experiments. The two traces are shown in Fig. 3, where the direct ${ }^{1} J_{\mathrm{HC}}$ correlation between $\mathrm{H}-1$ ' and $\mathrm{C}-1$ ' is shown together with the long-range ${ }^{3} J_{\mathrm{HC}}$ correlation between $\mathrm{H}-1^{\prime}$ and C-4' (central multiplet), since $\mathrm{C}-1^{\prime}$ and $\mathrm{C}-4^{\prime}$ have virtually identical chemical shifts. Traces I and II correspond to the application of sequences $1 \mathrm{~B}$ and $1 \mathrm{C}$, respectively. As expected, the signal intensity is doubled when applying pulse sequence $1 \mathrm{C}$, whereas the noise is increased by a factor of $\sqrt{2}$ (see Fig. 3 ). The resulting experimental $S / N$ is almost identical to the theoretical value $(62 / 44 \approx 1.41)$.

Additionally, one should appreciate that sequence $1 \mathrm{C}$ contains fewer pulses than 1B, and therefore it is less sensitive to RF pulse imperfections.

Finally, it is important to point out that the application of the method developed by Rance and Lewis (24-26), although convenient in many experiments, can hardly be expected to 


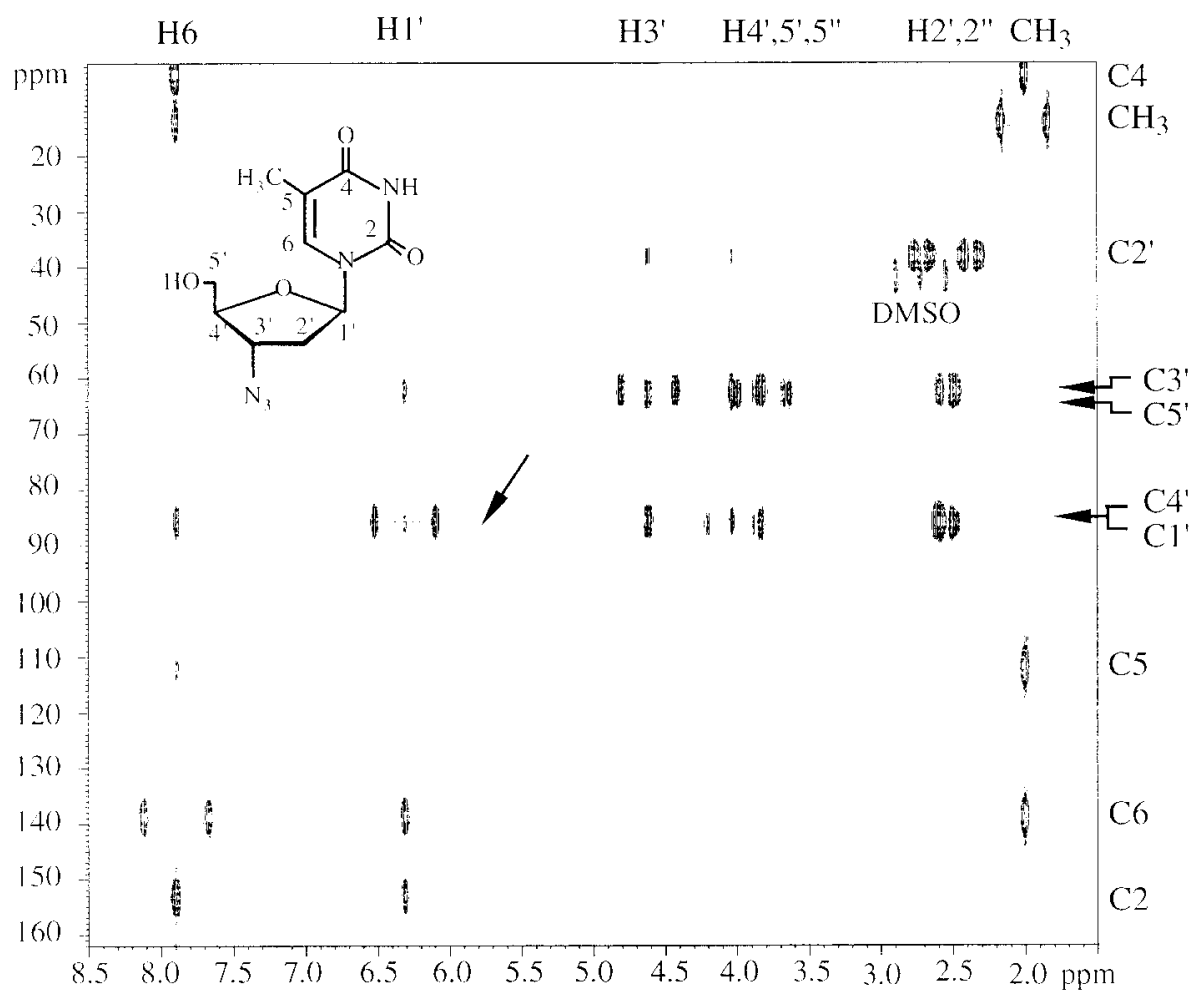

FIG. 2. 2D HMBC spectrum of a $50 \mathrm{mM} 3^{\prime}$-azido-3'-deoxythymidine (AZT) sample in DMSO- $d_{6}$, recorded at $500 \mathrm{MHz}$ and $25^{\circ} \mathrm{C}$ on a Bruker Avance spectrometer equipped with a $z$-shielded gradient triple-resonance probe, and using the pulse sequence $1 \mathrm{C}$. The delay $\Delta$ was set to 48.28 ms and the acquisition times $t_{2}$ and $t_{1}$ were $1.3 \mathrm{~s}$ (spectral width $6410 \mathrm{~Hz}, 8 \mathrm{~K}$ complex data points) and $3 \mathrm{~ms}$ (spectral width $20,100 \mathrm{~Hz}, 64$ real data points), respectively. The relaxation delay was $2 \mathrm{~s} ; 80$ scans were accumulated per $t_{1}$ increment. Sine-bell gradient pulses of $1 \mathrm{~ms}$ duration were used; $z$ gradients $G_{1}$ and $G_{2}$ were 25 and $15 \mathrm{G} / \mathrm{cm}$, respectively. The total acquisition time was approximately $6 \mathrm{~h}$. The arrow indicates the trace used for sensitivity comparison in Fig. 3 . C-4 is folded in the present experiment.

improve sensitivity in this particular case since it requires the refocusing of the corresponding heteronuclear coupling constants. This is perfectly feasible only for IS spin systems leading to a gain in $S / N$ of 2 compared to sequences like $1 \mathrm{C}$,
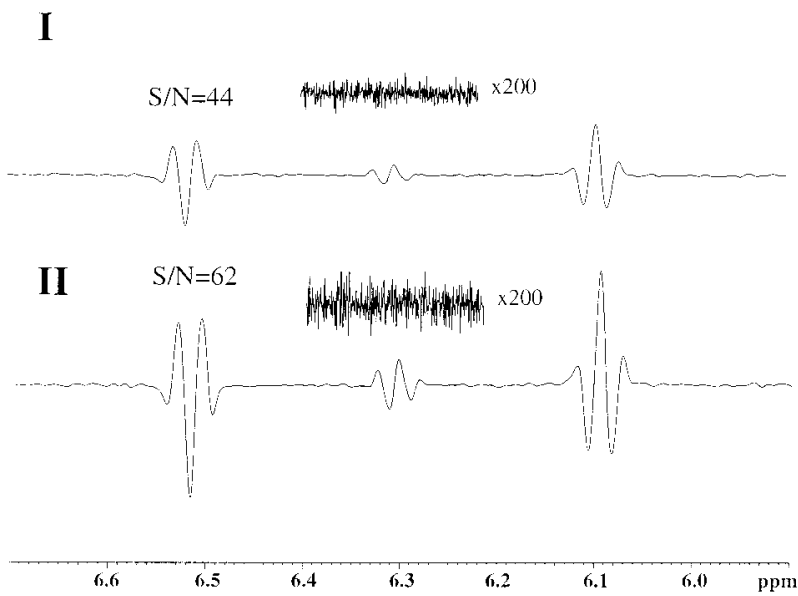

FIG. 3. Rows of the 2D experiments performed using sequences $1 \mathrm{~B}$ (trace I) and 1C (trace II). The rows were obtained at the chemical shift of carbons $1^{\prime}$ and $4^{\prime}$ (see Fig. 2). Both experiments were performed using identical conditions. or $2 \sqrt{2}$ with respect to sequence $1 \mathrm{~B}$. However, a fast inspection of Fig. 2 will suffice to realize that most of the spin systems are of the $\mathrm{I}_{n} \mathrm{~S}$ type, with $n$ ranging from 2 to 6 , or even more, since in this case $n$ represents the number of protons that are one, two, or three bounds removed from a given carbon. An extra complication comes from the fact that all the coupling constants are in principle different, spanning values as different as 1 to $11 \mathrm{~Hz}$. Clearly, as shown by Griesinger and co-workers (27) the refocusing is still at least partially feasible for systems like the directly coupled $\mathrm{CH}, \mathrm{CH}_{2}$, and $\mathrm{CH}_{3}$ groups, with the direct coupling constants of similar known magnitude. For long-range unknown coupling constants simply the trick does not apply.

In conclusion, the only possible sensitivity enhancement of the experiment proposed by Sheng and van Halbeek (12) is represented by our scheme (sequence 1C). One should appreciate that the echo-antiecho quadrature detection is here implemented by employing only two gradients, whereas three gradients are commonly used. The difference here is that with two gradients one needs, in alternate scans, to swap the gradient absolute intensities while keeping the same sign (see the legend to Fig. 1), whereas with three gradients one need just reverse the sign of one. In this case with a marginally more 
articulate use of just two gradients one avoids the introduction of a third one that would necessarily impose an extra delay. Therefore also in other pulse sequences one could find the use of our scheme more convenient than the conventional one.

A detailed description of the experiments, with particular attention to the coherence evolution induced by gradients, is reported in the Appendix, using the product operator formalism (28).

\section{APPENDIX}

Let us consider a three-spin system consisting of two protons $I_{1}$ and $I_{2}$, and a carbon-13 nucleus $S$, where the two protons are coupled with a coupling of $J_{\mathrm{H}}, \mathrm{I}_{1}$ is coupled to $\mathrm{S}$ with a coupling $J_{\text {IS }}$, and $\mathrm{I}_{2}$ is not coupled to $\mathrm{S}$. This system is sufficient to illustrate the phase modulation and the relative sensitivity of the sequences depicted in Fig. 1.

\section{Sequence $1 B$}

The first part of the sequence is identical to that already described by Titman et al. (18), with quadrature detection in $t_{1}$ by changing phase $\phi_{1}$. At point $a$ the magnetization can be described by (18)

$$
\begin{aligned}
\rho_{a}=\sin ( & \left.\pi J_{\mathrm{IS}} \Delta\right) \cos \left(\Omega_{\mathrm{S}} t_{1}\right)\left[-c_{\mathrm{H}} c_{1} 2 I_{1 x} S_{z}-c_{\mathrm{H}} s_{1} 2 I_{1 y} S_{z}\right. \\
+ & \left.s_{\mathrm{H}} s_{1} 4 I_{1 x} I_{2 z} S_{z}-s_{\mathrm{H}} c_{1} 4 I_{1 y} I_{2 z} S_{z}\right],
\end{aligned}
$$

where

$$
\begin{array}{ll}
c_{\mathrm{H}}=\cos \left(\pi J_{\mathrm{H}} \Delta\right), & s_{\mathrm{H}}=\sin \left(\pi J_{\mathrm{H}} \Delta\right), \\
c_{1}=\cos \left(\Omega_{1} \Delta\right), & s_{1}=\sin \left(\Omega_{1} \Delta\right) .
\end{array}
$$

Next, we apply the gradient selection. We can derive the expression for each one of the four terms in [1], and we will calculate the gradient refocusing condition by obtaining the expression for the first term $\left(2 I_{1 x} S_{z}\right)$. This term is converted by the subsequent pulses and gradients to

$$
\begin{array}{r}
-\frac{i}{2}\left[I_{1}^{+} S^{-} \exp \left(-i \theta_{a}\right)-I_{1}^{+} S^{+} \exp \left(-i \theta_{b}\right)\right. \\
\left.+I_{1}^{-} S^{-} \exp \left(i \theta_{b}\right)-I_{1}^{-} S^{+} \exp \left(i \theta_{a}\right)\right],
\end{array}
$$

where

$$
\begin{aligned}
& \theta_{a}=\gamma_{1} G_{1}+\left(\gamma_{\mathrm{I}}+\gamma_{\mathrm{S}}\right) G_{2}+\left(\gamma_{\mathrm{I}}-\gamma_{\mathrm{S}}\right) G_{3}, \\
& \theta_{b}=\gamma_{\mathrm{I}} G_{1}+\left(\gamma_{\mathrm{I}}-\gamma_{\mathrm{S}}\right) G_{2}+\left(\gamma_{\mathrm{I}}+\gamma_{\mathrm{S}}\right) G_{3}
\end{aligned}
$$

$G_{i}=B_{1(z) i} \tau_{i}$ is the product of the gradient strength by the gradient duration, and $\gamma_{\mathrm{I}}$ and $\gamma_{\mathrm{S}}$ are the gyromagnetic ratios of proton and carbon, respectively. Then one can choose a gra- dient combination that makes either $\theta_{a}$ or $\theta_{b}$ zero (but not both). With $\theta_{a}=0$ one can deduce the following transformation produced by the gradient selection:

$$
2 I_{x} S_{z} \rightarrow-\frac{1}{2}\left(2 I_{x} S_{z}\right)
$$

A similar result can be deduced for the other three terms within [1], yielding the following expressions for the two separate experiments:

odd experiments $\left(\phi_{1}=x\right)$ :

$$
\frac{1}{2} \sin \left(\pi J_{\mathrm{IS}} \Delta\right) \cos \left(\Omega_{\mathrm{S}} t_{1}\right)[A]
$$

even experiments $\left(\phi_{1}=y\right)$ :

$$
\frac{1}{2} \sin \left(\pi J_{\mathrm{IS}} \Delta\right) \sin \left(\Omega_{\mathrm{S}} t_{1}\right)[A]
$$

where

$$
\begin{aligned}
A= & -c_{\mathrm{H}} c_{1} 2 I_{1 x} S_{z}-c_{\mathrm{H}} s_{1} 2 I_{1 y} S_{z} \\
& +s_{\mathrm{H}} s_{1} 4 I_{1 x} I_{2 z} S_{z}-s_{\mathrm{H}} c_{1} 4 I_{1 y} I_{2 z} S_{z} .
\end{aligned}
$$

Cross peaks are obtained with an absorption-mode lineshape in $F_{1}$ (the cosine- and sine-modulated terms are separate), and with a complex superposition of lines of all phases in $F_{2}$. If compared to the standard quadrature-detected experiments without the use of gradients a factor of 2 in sensitivity is formally lost.

\section{Sequence $1 C$}

At point $a$ the density operator has the form

$$
\begin{aligned}
\rho_{a}=\sin & \left(\pi J_{\mathrm{IS}} \Delta\right) \cos \left(\Omega_{\mathrm{S}} t_{1}\right)\left[-c_{\mathrm{H}} c_{\mathrm{I}} 2 I_{1 x} S_{y}-c_{\mathrm{H}} s_{1} 2 I_{1 y} S_{y}\right. \\
& \left.+s_{\mathrm{H}} s_{1} 4 I_{1 x} I_{2 z} S_{y}-s_{\mathrm{H}} c_{1} 4 I_{1 y} I_{2 z} S_{y}\right] \\
- & \sin \left(\pi J_{\mathrm{IS}} \Delta\right) \sin \left(\Omega_{\mathrm{S}} t_{1}\right)\left[-c_{\mathrm{H}} c_{1} 2 I_{1 x} S_{x}-c_{\mathrm{H}} s_{1} 2 I_{1 y} S_{x}\right. \\
& \left.+s_{\mathrm{H}} s_{1} 4 I_{1 x} I_{2 z} S_{x}-s_{\mathrm{H}} c_{1} 4 I_{1 y} I_{2 z} S_{x}\right] .
\end{aligned}
$$

As in the precedent case, we will derive the expression at point $b$ for the first term of each bracket in [6]. It can be easily calculated that these two terms are transformed into

$$
\begin{array}{r}
2 I_{1 x} S_{y} \rightarrow \frac{1}{2 i}\left[I_{1}^{+} S^{-} \exp \left(-i \theta_{1}\right)-I_{1}^{+} S^{+} \exp \left(-i \theta_{2}\right)\right. \\
\left.\quad+I_{1}^{-} S^{-} \exp \left(i \theta_{2}\right)-I_{1}^{-} S^{+} \exp \left(i \theta_{1}\right)\right] \\
2 I_{1 x} S_{x} \rightarrow \frac{1}{2}\left[I_{1}^{+} S^{-} \exp \left(-i \theta_{1}\right)+I_{1}^{+} S^{+} \exp \left(-i \theta_{2}\right)\right. \\
\left.+I_{1}^{-} S^{-} \exp \left(i \theta_{2}\right)+I_{1}^{-} S^{+} \exp \left(i \theta_{1}\right)\right]
\end{array}
$$


where

$$
\begin{aligned}
& \theta_{1}=\left(\gamma_{\mathrm{I}}+\gamma_{\mathrm{S}}\right) G_{1}+\left(\gamma_{\mathrm{I}}-\gamma_{\mathrm{S}}\right) G_{2}, \\
& \theta_{2}=\left(\gamma_{\mathrm{I}}-\gamma_{\mathrm{S}}\right) G_{1}+\left(\gamma_{\mathrm{I}}+\gamma_{\mathrm{S}}\right) G_{2}
\end{aligned}
$$

In this case we will perform two separate experiments. The odd experiments will have a ratio $G_{1}: G_{2}$ so as to make $\theta_{1}=0$. The even experiments will make $\theta_{2}=0$. This can be readily achieved by using the following gradient ratios:

$$
\begin{aligned}
\text { odd experiments: } & G_{1}: G_{2}=5:-3 \\
\text { even experiments: } & G_{1}: G_{2}=3:-5 .
\end{aligned}
$$

Using these two conditions, we can calculate the final form of these two operators in both experiments, just before detection:

odd experiments $\left(\theta_{1}=0\right)$ :

$$
2 I_{1 x} S_{y} \rightarrow-\frac{1}{2}\left(2 I_{1 x} S_{z}\right), \quad 2 I_{1 x} S_{x} \rightarrow-\frac{1}{2}\left(2 I_{1 y} S_{z}\right)
$$

even experiments $\left(\theta_{2}=0\right)$ :

$$
2 I_{1 x} S_{y} \rightarrow-\frac{1}{2}\left(2 I_{1 x} S_{z}\right), \quad 2 I_{1 x} S_{x} \rightarrow+\frac{1}{2}\left(2 I_{1 y} S_{z}\right)
$$

This result can be extended to the remaining terms in [6], producing the following expression for the observable magnetization:

odd experiments $\left(\theta_{1}=0\right)$ :

$$
-\frac{1}{2} \sin \left(\pi J_{\mathrm{IS}} \Delta\right) \cos \left(\Omega_{\mathrm{S}} t_{1}\right)[A]+\frac{1}{2} \sin \left(\pi J_{\mathrm{IS}} \Delta\right) \sin \left(\Omega_{\mathrm{S}} t_{1}\right)[\bar{A}]
$$

even experiments $\left(\theta_{2}=0\right)$ :

$$
-\frac{1}{2} \sin \left(\pi J_{\mathrm{IS}} \Delta\right) \cos \left(\Omega_{\mathrm{S}} t_{1}\right)[A]-\frac{1}{2} \sin \left(\pi J_{\mathrm{IS}} \Delta\right) \sin \left(\Omega_{\mathrm{S}} t_{1}\right)[\bar{A}],
$$

where $A$ has the same meaning as in [4], and $\bar{A}$ represents the same operators but with a $90^{\circ}$ phase difference for $I_{1}$.

As already described (26), the processing recipe that produces a phase-sensitive spectrum implies the sum and the difference of the two experiments and $\mathrm{a} 90^{\circ}$ phase correction of each second experiment, to yield

$$
\begin{array}{ll}
\sin \left(\pi J_{\mathrm{IS}} \Delta\right) \cos \left(\Omega_{\mathrm{S}} t_{1}\right)[A] & (\text { sum }) \\
\sin \left(\pi J_{\mathrm{IS}} \Delta\right) \sin \left(\Omega_{\mathrm{S}} t_{1}\right)[A] & \text { (difference). }
\end{array}
$$

By comparing [13] with [4] and [14] with [5], one can easily recognize the gain factor of 2 in signal intensity, although, as we have obtained this result by combining two experiments, the noise is also increased by a factor of $\sqrt{2}$. In conclusion, the final gain in signal/noise is, as it turns out, precisely $\sqrt{2}$.

\section{REFERENCES}

1. J. L. Marshall, "Carbon-Carbon and Carbon-Proton NMR Couplings: Application to Organic Stereochemistry and Conformational Analysis," Verlag Chemie International, Florida (1983).

2. D. O. Cicero, G. Barbato, and R. Bazzo, Tetrahedron 51, 1030310316 (1995).

3. W. Kozminski and D. Nanz, J. Magn. Reson. 142, 294-299 (2000).

4. W. Kozminski and D. Nanz, J. Magn. Reson. 124, 383-392 (1997).

5. D. Uhrin, G. Batta, V. J. Hruby, P. N. Barlow, and K. E. Kover, J. Magn. Reson. 130, 155-161 (1998).

6. M. Kurtz, P. Schmieder, and H. Kessler, Angew. Chem. Int. Ed. Engl. 30, 1329-1331 (1991).

7. R. T. Williamson, B. L. Marquez, W. H. Gerwick, and K. E. Kover, Magn. Reson. Chem. 38, 265-273 (2000).

8. R. Marek, L. Kralik, and V. Sklenar, Tetrahedron Lett. 38, 665- 668 (1997).

9. R. Marek, Collect. Czech. Chem. Commun. 62, 1747-1753 (1997).

10. K. Furihata and H. Seto, Tetrahedron Lett. 40, 6271-6275 (1999).

11. G. Zhu, A. Renwick, and A. Bax, J. Magn. Reson. 110, 257-261 (1994).

12. S. Sheng and H. van Halbeek, J. Magn. Reson. 130, 296-299 (1998).

13. W. Wilker and D. Leibfritz, Magn. Reson. Chem. 33, 632-638 (1995).

14. K. Ding, Magn. Reson. Chem. 38, 321-323 (2000).

15. C. H. Gotfresen, A. Meissner, J. O. Duus, and O. W. Sørensen, Magn. Reson. Chem. 38, 692-695 (2000).

16. R. Bazzo, G. Barbato, and D. O. Cicero, J. Magn. Reson. A 117, 267-271 (1995).

17. A. Bax and M. F. Summers, J. Am. Chem. Soc. 108, 2093-2094 (1986).

18. J. J. Titman, D. Neuhaus, and J. Keeler, J. Magn. Reson. 85, 111-131 (1989).

19. J. M. Richardson, J J . Titman, J. Keeler, and D. Neuhaus, J. Magn. Reson. 93, 533-553 (1991).

20. R. E. Hurd, B. K. John, and H. D. Plant, J. Magn. Reson. 93, $666-670$ (1991).

21. G. W. Vuister, J. Ruiz-Cabello, and P. C. M. van Zijl, J. Magn. Reson. 100, 215-220 (1992).

22. A. L. Davis, J. Keeler, E. D. Laue, and D. Moskau, J. Magn. Reson. 98, 207-216 (1992).

23. J . Schleucher, M. Sattler, and C. Griesinger, Angew. Chem. Int. Ed. Engl. 32, 1489-1491 (1993).

24. J . Cavanagh, A. G. Palmer, III, P. E. Wright, and M. Rance, J . Magn. Reson. 91, 429- 436 (1991).

25. A. G. Palmer, III, J . Cavanagh, P. E. Wright, and M. Rance, J . Magn. Reson. 93, 151-170 (1991).

26. L. E. Kay, P. Keifer, and T. Saarinen, J. Am. Chem. Soc. 114, 10663-10665 (1992).

27. J. Schleucher, M. Schwendinger, M. Sattler, P. Schmidt, 0. Schedletzky, S. J. Glaser, O. W. Sørensen, and C. Griesinger, J. Biomol. NMR 4, 301-306 (1994).

28. O. W. Sørensen, G. W. Eich, M. H. Levitt, G. Bodenhausen, and R. R. Ernst, Prog. NMR Spectrosc. 16, 163 (1983).

29. D. Marion, M. Ikura, R. Tschudin, and A. Bax, J. Magn. Reson. 85, 393-399 (1989). 